\title{
Using SIR Model to Simulate Emotion Contagion in Dynamic Crowd Aggregation Process
}

\author{
Nan Xianga,, Zehong Zhou ${ }^{\mathrm{a}}$, Zhigeng Pan ${ }^{\mathrm{b}}$ \\ ${ }^{a}$ Liangjiang International College, Chongqing University of Technology, Chongqing, 401135, China \\ ${ }^{b}$ Institute of Service Engineering, Hangzhou Normal University, Hangzhou, 311121, China
}

\begin{abstract}
Emotion contagion is an indispensable behavior in a dynamic crowd, especially in an evacuation situation. As a consequence, generating emotion contagion results is very useful in the crowd simulation field. However, because the topology of the crowd usually keeps changing dynamically, computing the contagion process is a challenge. In this paper, we represented our research about the emotion contagion effects on the virtual pedestrian dynamic aggregation process. First of all, we calculated individuals' moving parameters based on their prefixed expectations according to the social force theory. After this, we made an adjacent test for each individual to generate nearer neighbors for further emotional contagions computing between neighbors. We then treated the emotional contagions between individuals and their neighbors as the information spreading process so that we can adopt the emotional information spreading model SIR (Susceptible Infective Removal) to calculate emotional influences, which are represented as their changing moving velocities during aggregation. Social force for computing low level moving parameters and SIR model for generating emotional influences were integrated by our method to simulate the dynamic pedestrian aggregation. Experimental results showed that the SIR model can effectively improve the fidelity of the emotional interaction process and crowd aggregation.
\end{abstract}

Keywords: pedestrian simulation; dynamic crowd aggregation; emotional contagion; information spreading; SIR model

(Submitted on November 20, 2017; Revised on December 10, 2017; Accepted on December 23, 2017)

() 2018 Totem Publisher, Inc. All rights reserved.

\section{Introduction}

Crowd aggregation is an important representation of pedestrian group behaviors and has gradually become a hot research point in the virtual crowd simulation field. The traditional crowd simulations mainly contained low level or physical level problems, such as path planning and collision avoidance [8,19]. For example, in the traffic monitoring application, the existing systems can estimate the crowd density according to perceived number of pedestrians [23]. However, the dynamic aggregation process was not deeply studied. Another application case was in evacuation situations. According to the building structure analysis information, the relevant models [2,18,26] based on the finite element theory can calculate the bottleneck areas for building designers to optimize the building architecture. But the analysis process based on finite element was very complex and was not suitable for aggregation animation. As a consequence, modeling the dynamic pedestrians, especially the emotional interactions between the individuals, gradually attracted the attention of scholars [3,6]. However, the existing emotion contagion models only constructed simple emotional propagating models between individuals of the crowd [20]. The time dependent changes of emotional influences should be taken into account [16].

In this paper, we provided a model for simulating the dynamic crowd aggregation process. We simulated the aggregation of outdoor pedestrians, represented by the influences from emotional contagion to pedestrian aggregation. In addition, we adopted the social force theory to calculate the velocities of the individuals, as well as the SIR model to generate the individual emotional affections in the crowd. Furthermore, in our research, the emotional contagion between individuals will be represented as individual moving parameter changing, including the speed and direction of velocity. As a consequence, these changing parameters further influenced crowd aggregation. To reduce the time consumption of the

\footnotetext{
* Corresponding author.

E-mail address: xiangnan@cqut.edu.cn
} 
aggregation model, we represented the crowd emotion propagating process as the individual desired velocity propagating. Most importantly, inspired by researches on the information communication field, we exploited the SIR model to simulate the emotional contagion process, as the emotion contagion between two individuals could be treated as a type of information spreading. Then, due to the SIR model, the time dependent changes of individuals' affectations could be well simulated.

\section{Related Work}

In the crowd simulation field, the social force model, cellular automata (CA) model and agent based model were common microscopic models, which were often used in crowd evacuation simulation. Zhao [25] used the cellular automaton model to simulate panic evacuation behaviors and discussed the effects of population density on aggregation. Their study found that the crowd gathered quickly when the indoor population density was low under the influence of the repulsive force. Papadopoulos [15] added slime-like self-protection molds in the cellular automaton to simulate the behavior of the crowd and provided a quantitative analysis method that used a velocity-density diagram and flow-density diagram. Compared with the discrete cells, social forces can simulate the continuous movement of objects, and Zeng [22] adopted it to simulate the behaviors of the crowd crossing the road. Yang [21] used the improved social force model to simulate a crowd that had a leader. Current researchers have shown that the models based on the social force model can effectively simulate the moving characteristics of various types of crowds. Although the models based on the social force were relatively simple, the individuals within the crowd were lacking in intelligence, which promoted the development of agent based simulations. Tan [18] increased knowledge about evacuation sites for the agent and simulated the escape processes at different levels of knowledge. In the research of Martinez [9], the intelligence of agents had been further embodied. It used the reinforcement learning theory to make the agent learn the knowledge of the scene independently, so as to select the effective way to evacuate.

In the affective computing part, agent emotional affections were also import to crowd simulation in addition to the agent intelligence. Emotion contagion in crowd simulation has been studied for many years and has been applied in situations such as escape panic [2]. Xiang [20] exploited the GPU accelerated heat conduction model to generate the emotion contagion results; however, the results were not accurate in the dynamic crowd. Appraisal theory [5] was a wildly used theory within emotional affection computing, and served as the basis for several computational models of emotion, as it had the special feature domain-independence. However, considering the time consumption in crowd simulation field, the agents were too dense to adopt such theory. Bosse [1] used an agent-based approach to formalize and simulate emotion contagion processes within groups, which may involve absorption or amplification of emotions of others. They defined a sender-channel-receiver architecture to compute the contagion results. In this paper, we treated the emotion contagion as the information spreading so that we could exploit the current information spreading models.

Most of the studies on information spreading stemmed from the classical SIS (Susceptible Infected Susceptible) model and SIR (Susceptible Infective Removal) model, such as the earlier DK (Daley Kendal) and MK (Maki Thomson) rumor propagation model [24]. Mereno [10] and Nekovee [12] developed the DK rumor propagation model, proposed by Daley, by integrating the mean field equation in the homogeneous network and the non-uniform network. Prakash [14] provided theory and immunization algorithms about virus propagation on time-varying networks, which constructed a foundation for further information spreading model improvements. Navlakha [11] proposed a new generative model of network evolution in dynamic and harsh environments. Their model can reproduce the range of topologies observed across known robust and fragile biological networks, as well as several additional transport, communication, and social networks. Ojugo [13] studied the logarithmic analysis of complex networks yields solution to help minimize virus spread and propagation over networks. Sanatkar [17] derived an epidemic threshold, considering the susceptible-infected-susceptible epidemic model. In their research, an epidemic probabilistic model was developed assuming independence between states of nodes, and the conditions were identified under which the epidemic died out by linearizing the underlying dynamical system and analyzing its asymptotic stability around the origin. However, these traditional propagation models were either too simple or too difficult to be used in emotion contagion. So, we must provide an easy way for the information spreading model application.

\section{Simulation Pipeline}

In this part, we mapped the 3D crowds into 2D space to reduce time consumption. Similar to the collision detection method, as shown in Figure 1, the individuals first selected the moving target and generated the initial expected speed; the individuals were secondly projected onto two-dimensional ground and represented by squares; thirdly, an adjacent squares test was made to generate the nearer neighbors and calculate the contagion results based on SIR model. 


\subsection{Pedestrian Force based Velocity Generation}

When in a disaster evacuation situation, individuals were affected by both social psychology and the physical environment as shown in equal (1).

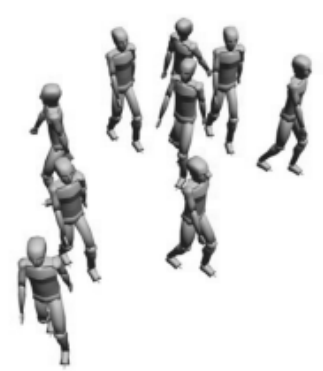

Projection \&

Velocity Generation
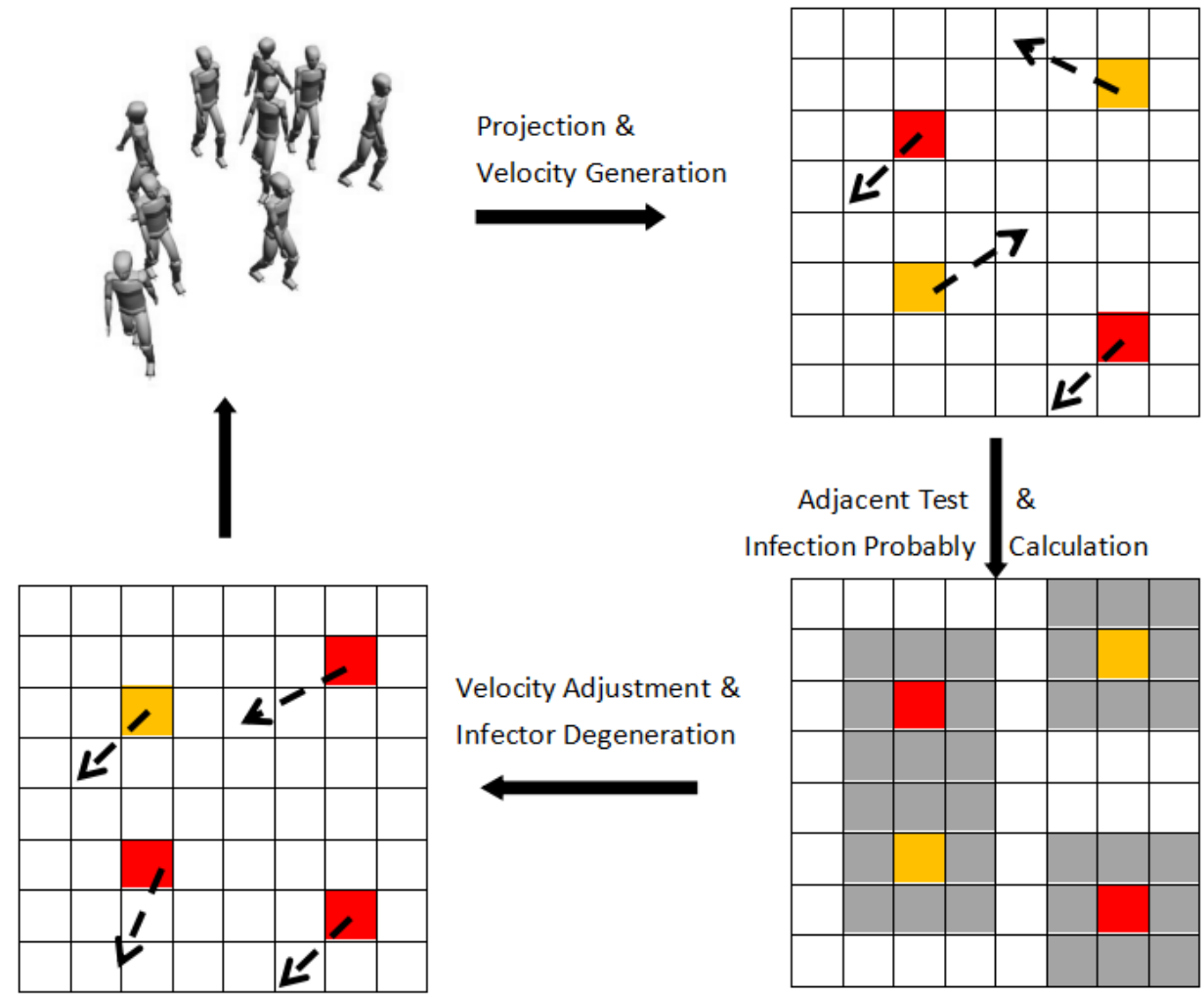

Adjacent Test

\& Infection Probably Calculation

Velocity Adjustment \& Infector Degeneration
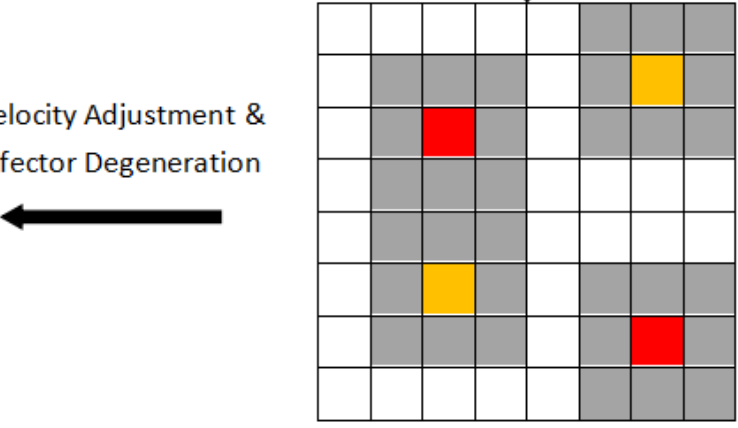

Figure 1. Processing pipeline

$$
m_{i} \frac{\mathrm{d} v_{i}}{\mathrm{~d} t}(t)=m_{i} \frac{v_{i}^{0} e_{i}^{0}-v_{i}}{\tau_{i}}+\sum_{j(\neq i)} f_{i j}+\sum_{w} f_{i w}
$$

Where $m_{i}$ was individual mass, $\mathrm{v}^{0}{ }_{i}$ was expected speed value and $e^{0}{ }_{i}$ denoted expected direction. $v_{i}$ was current velocity, and $\tau_{i}$ denoted force duration. The latter two items were the sum of the individual's influences by other individuals $\sum_{j \neq i} f_{i j}$ and the influences of the obstacle on the individual $\sum_{w} f_{i w}$ can be calculated in formula (2).

$$
\left\{\begin{array}{l}
f_{i j}=f_{i j n} n_{i j}+f_{i j t} t_{i j} \\
f_{i w}=f_{i w n} n_{i w}+f_{i w t} t_{i w}
\end{array}\right.
$$

$f_{i j n}, f_{i w n}$ represented normal components, $f_{i j t} 、 f_{i w t}$ represented tangential components, whose details can be found in reference [22].

\subsection{Affection Projection}

An individual could affect his or her neighbors by body movements, facial expressions or voices, and in our previous works[20], we used a cylinder to model his or her affection area and project the individual affection cylinder of $i$ onto two dimensional ground R, as shown in Figure 2. 
Here a $3 * 3$ square template $S_{i}{ }_{i}$ was adopted to simplify the simulation of the circular area. In this way, the red square was the individual's current position $\operatorname{Pos}_{i}{ }_{i}$, and the 8 adjacent squares represented his affection fields Aff ${ }_{i}$ at time $t$, as shown in Figure 2. We can easy draw a conclusion that $\operatorname{Pos}_{i}^{t} \cup A f f{ }_{i}{ }_{i}=S_{i}^{t}$. As a consequence, the individual could propagate their emotions to the individuals located in their 8 adjacent squares.

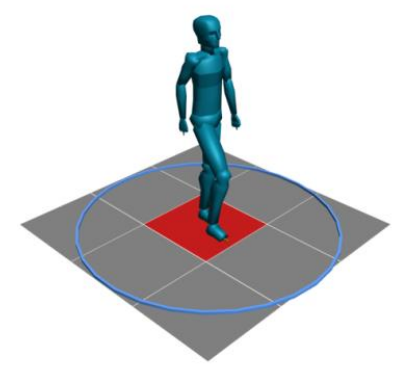

Figure 2. Affection projection

\subsection{Adjacent Individuals Test}

After we projected the crowd into squares, we needed to find the adjacent individuals for emotion contagion. This step could be formulated for an individual $i$, by checking whether $\exists j, \operatorname{Pos}_{j}^{t} \cap A f f_{i}^{t} \neq \phi$. We can find in Figure 3 that there were three adjacent cases for two squares in a two-dimensional space. In case one, two individuals were not adjacent but their affection areas were overlapping. In case two, two individuals were adjacent. Although their adjacent position relations could vary, we only presented one relation as an example. Similar to case two, we only presented one situation for case three, which represented two individuals who have no adjacent relations.
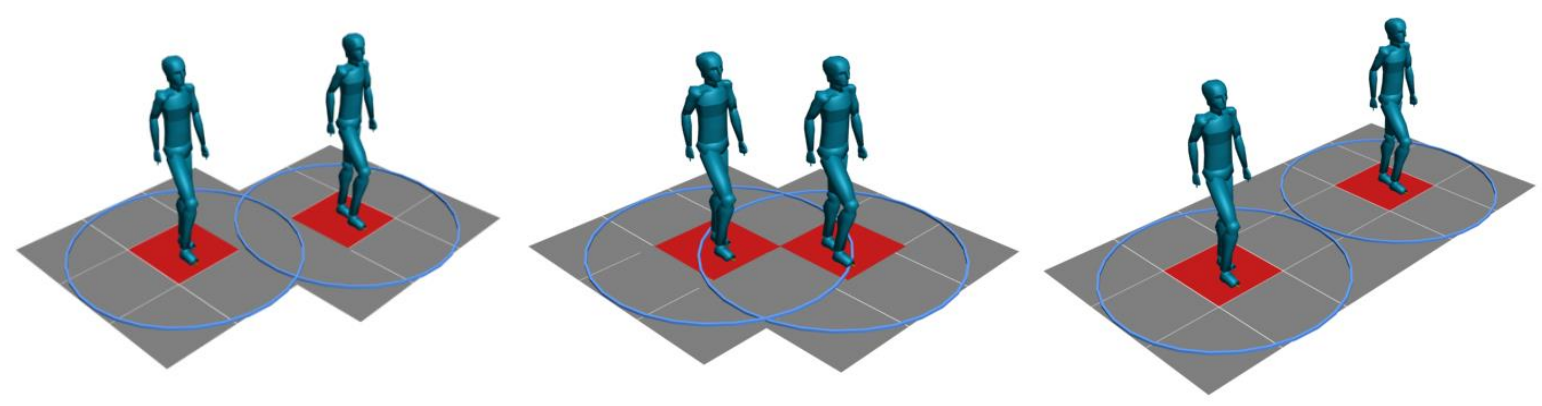

Figure 3. Relations between two individuals.

To quickly generate the adjacent results, a two-dimensional array $A O^{t}$ was adopted to store the occupation situations of the squares for the crowd. As the crowd moved dynamically, we created another array $A P^{t}$ to store the individual positions. This adjacent test process could be divided into the following steps: firstly, get individual $i$ 's position from position array $A P^{t}$; secondly, according to $i$ 's position, get the positions of adjacent individuals based on $A O^{t}$.

\subsection{Collision Avoidance}

Generating the moving parameters for the crowd was a foundation for representing the individuals. Within this step, collision avoidance was very essential. As we used a square to represent an individual's position, it is easy to calculate his or her next position based on social force. Frictional forces were not integrated into our model, as we focused on the emotional affections on individuals' velocities. We assumed the pedestrians were not moving fast, so the density was relatively higher. Let every individual have a target orientation $\operatorname{Dtar}_{i}^{t}$ and obey the following rules:

- The probability for an individual to choose target orientation $\operatorname{Dtar}_{i}^{t}$ as moving orientation $\operatorname{Dmov}^{t+0}{ }_{i}$ was $80 \%$; and to choose either side of the $\operatorname{Dtar}_{i}^{t}$ as $\operatorname{Dmov}^{t+0}{ }_{i}$ was $10 \%$.

- The probability for an individual to go through either side (Dsid $1^{t+0}{ }_{i} \operatorname{Dsid}^{t+0}{ }_{i}$ ) of the $\operatorname{Dmov}^{t+0}{ }_{i}$ was $50 \%$ when the next square in the $\operatorname{Dmov}^{t+0}{ }_{i}$ was not available. 
- If $D$ sid $1^{t+0}{ }_{i}$ and $D$ sid $2^{t+0}{ }_{i}$ were not available, individual should stay in current position.

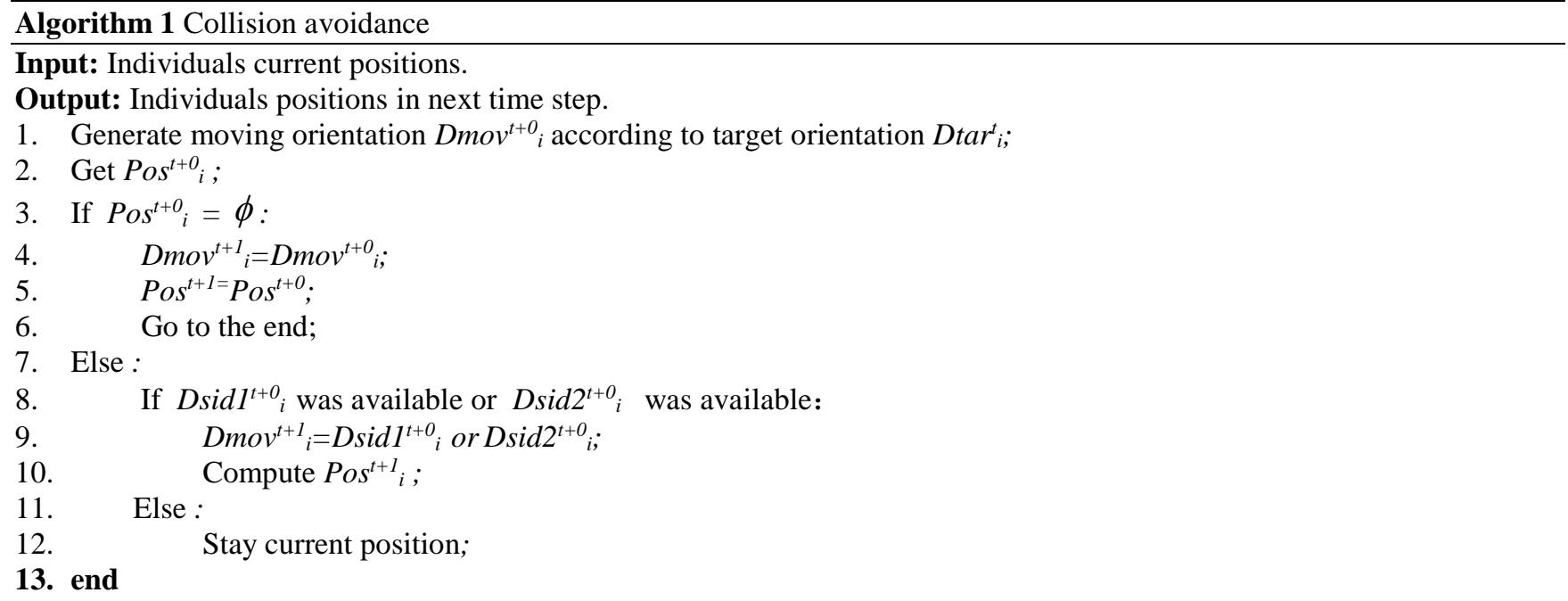

\subsection{SIR Based Emotion Contagion}

This model simplified the process of individual emotional interaction into the expected velocity transfer process, that was to set $|V| \propto \mathrm{A}$. Where $V$ denoted expected velocity and A represented emotional arousal level. Then, after emotion interaction in time $\mathrm{t}$, individual a's expected velocity in time $t+l$ can be calculated by formula (3).

$$
V_{a}^{t+1}=\left(1-\alpha_{a}\right) V_{a}^{t}+\alpha_{a} V_{b}^{t}
$$

Where $\alpha$ was the probability of individuals who had been affected. As we used the SIR based model to simulate emotion contagion, $\alpha$ denoted the probability that a susceptible transferred into a infector and could be generated by formula (4) reference by Meng's research[7]:

$$
\alpha=1-(1-\lambda)^{\omega \frac{\delta(i)}{d_{r}(i)}}
$$

Where $\lambda$ was the information spreading rate that reflected the impacts of the message itself. $\omega$ was control factor based on Centola's research[4], $\delta(i)$ denoted total amount of received information, $d_{r}(i)$ denoted immunization degree.

$$
\begin{gathered}
\delta(i)=\sum_{j \in a j s(i)} d_{a}(j) \\
d_{a}(i)=d_{r}(i)=\lg \left(k_{i}+1\right)
\end{gathered}
$$

ajs $(i)$ represented the neighbors, $d_{a}(i)$ represented authority level. Both of them were calculated by exponential function without degree $k_{i}$.

In most existing SIR models, information spreading occurred when individuals were contacting. However, the infector would degenerate into ignorant or immune during the contact process. In the classical rumor propagation model [10,12], the infector would change into immune with a fixed probability when exposed to other infectors or immune individuals. The probability $p_{d e}^{i}$ in time $t$, could be calculated by formula (7), where $\beta$ was the empirical parameter referenced to Nekovee's work, and $g(i, t)$ meant the sum of the infectors and immune individuals contacted with $i$ in time $t$.

$$
p_{d e}^{i}(t)=1-(1-\beta)^{g(i, t)}
$$


The state transfer process could be found in Figure 4.

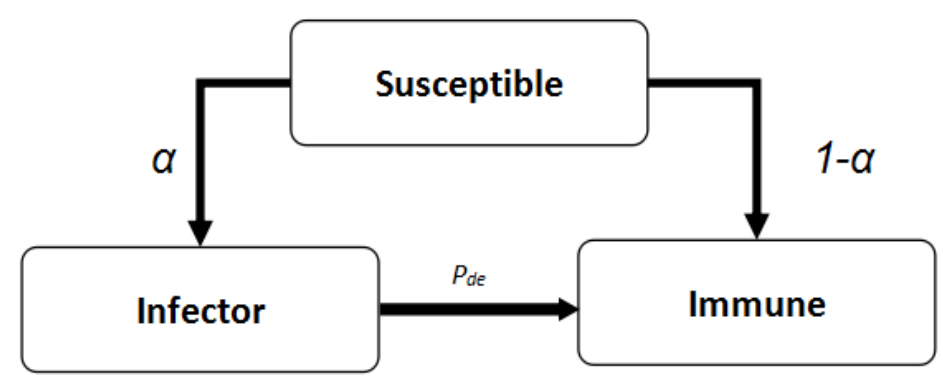

Figure 4. Individuals' states transfer map.

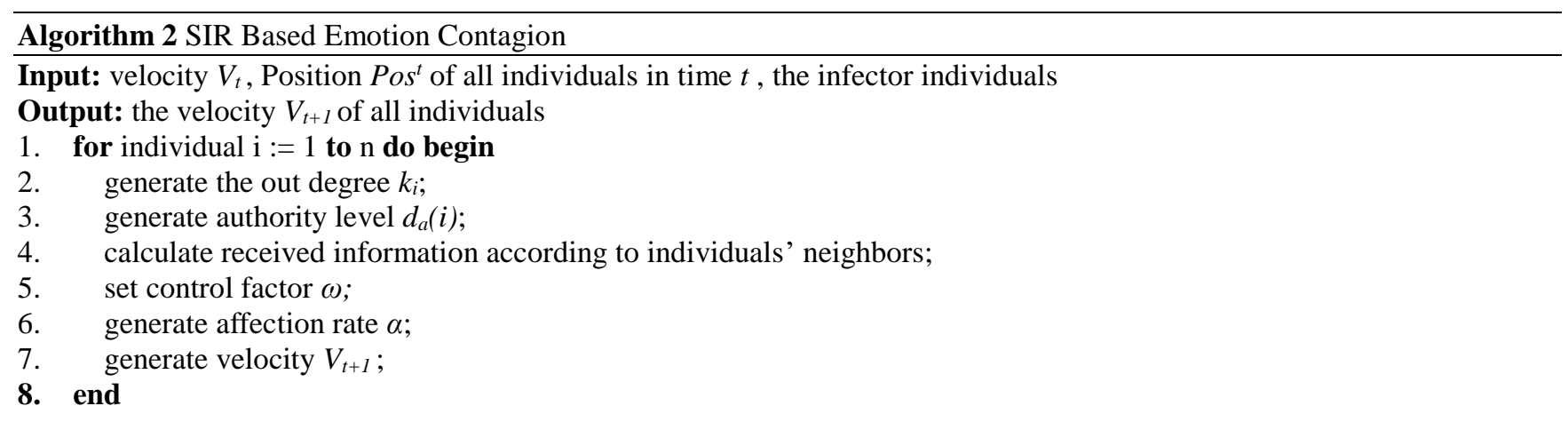

\section{Simulation Results \& Analysis}

We adopted Intel I5 CPU and Nvidia GTX770 GPU, which has 8 multiprocessors and 192 cores for each multiprocessor to run crowd simulation programs to test our algorithm. The average speed trace is shown in Figure 5, where we recorded some certain time points. Within this figure we can find that without emotional affections, the crowd's average speed was relatively stable from the beginning and decreased linearly. Compared with this situation, when a crowd moves with emotional affections the average speed increased sharply. As a consequence, the crowd arrived at the target quicker than the former one, and then their speeds decreased sharply as well.

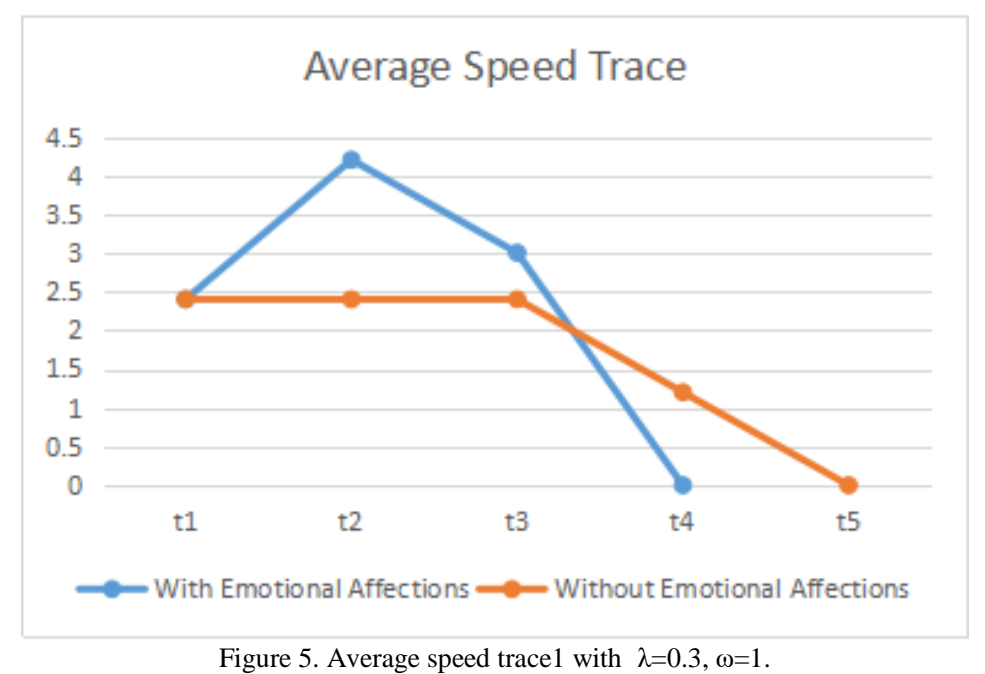

Figure 6 shows the off-line rendered emotion contagion simulation results on 400 individual aggregation processes with $\lambda=0.3, \omega=1$. We let the crowd move into the central red box that represented the source of infection. The nearer individuals were infected the first time, as shown in the top-right image. The individuals who got infected would move faster to the infection source. Then, these individuals became infectors and spread the emotional information to their neighbors as shown in the Figure 6 bottom images. Because the red individuals were infected, their expected velocities were changed. As a 
consequence, their density was relatively higher than those of blue ones, and the aggregation process was hastened. The compared aggregation without emotional affectations are shown in Figure 7.

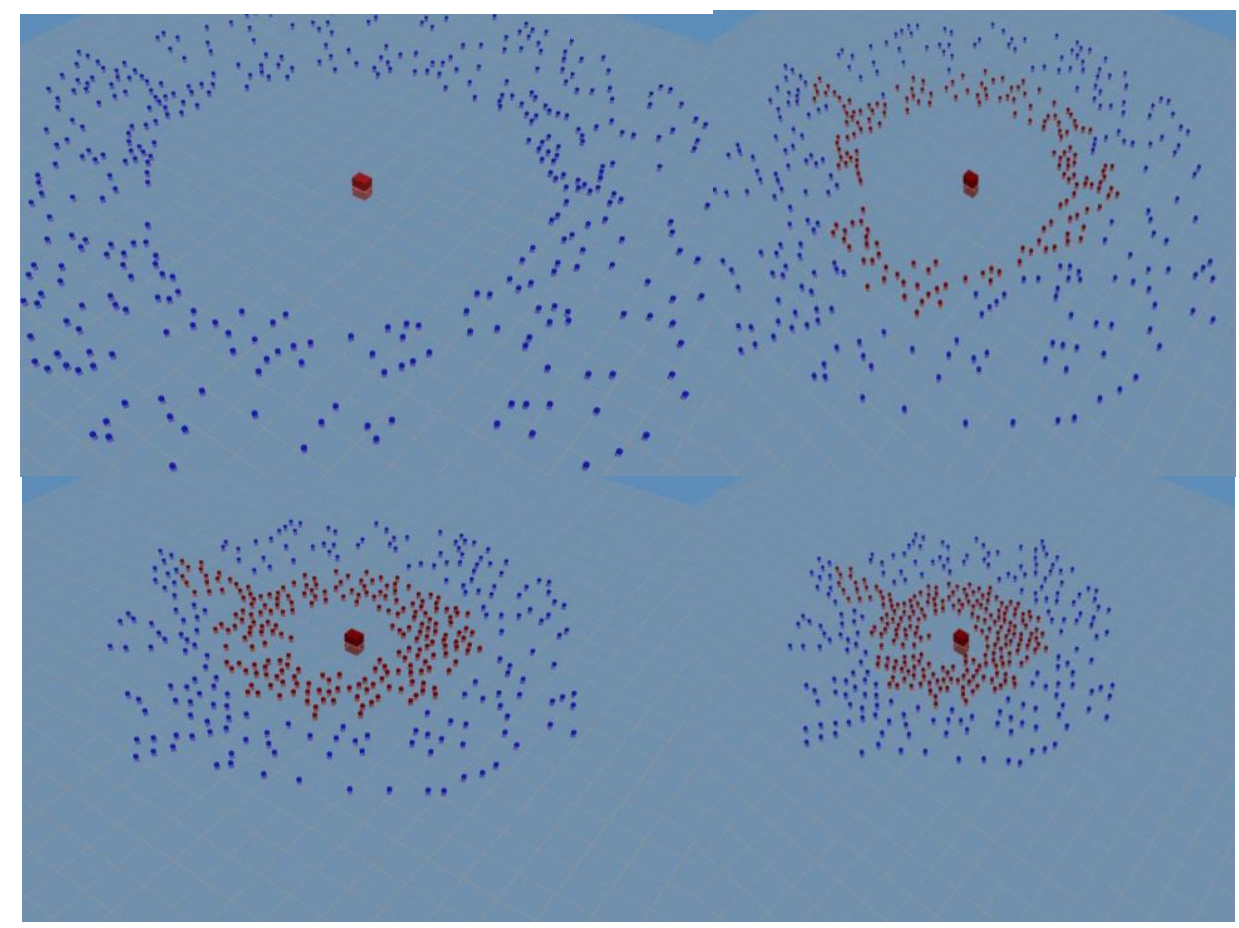

Figure 6. Simulation performances without infector degeneration.

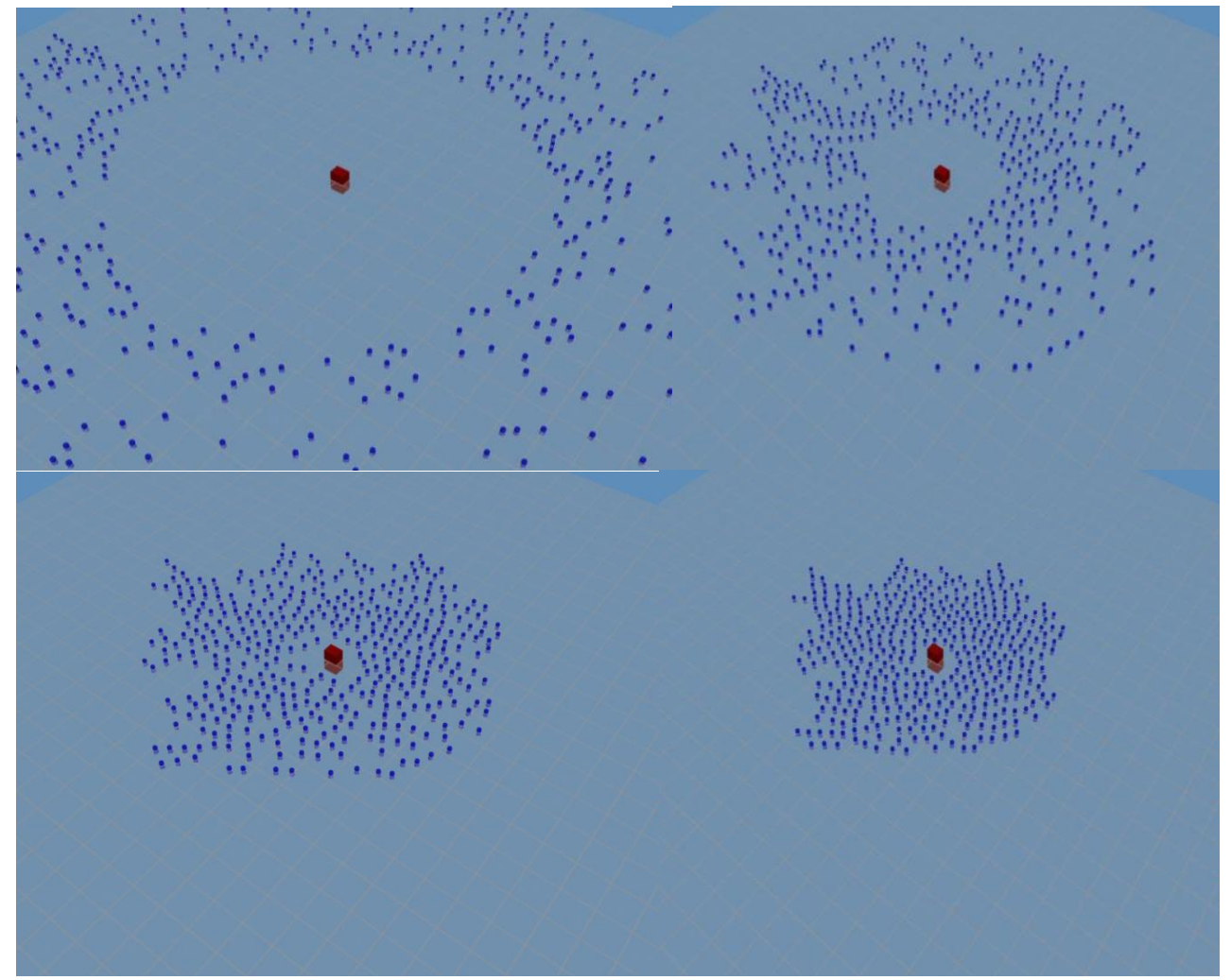

Figure 7. Simulation performances without infections.

As mentioned, the infectors would degenerate during emotion propagation. However, Figure 6 did not simulate this process. We represented degeneration in Figure 8. Here, we let red individuals be the infectors and the cyan individuals be 
the susceptible ones. The infectors' velocities pointed to the purple box and the others were pointed to the right end. During the emotion propagating process, some susceptible individuals were infected and moved to the purple box, and the others still maintained the original target, as shown in the bottom images. Compared with Figure 6, we can clearly see that some red individuals went to the right end, too. This represented the situation that infectors degenerated into immune ones. The compared results with no infections are shown in Figure 9.

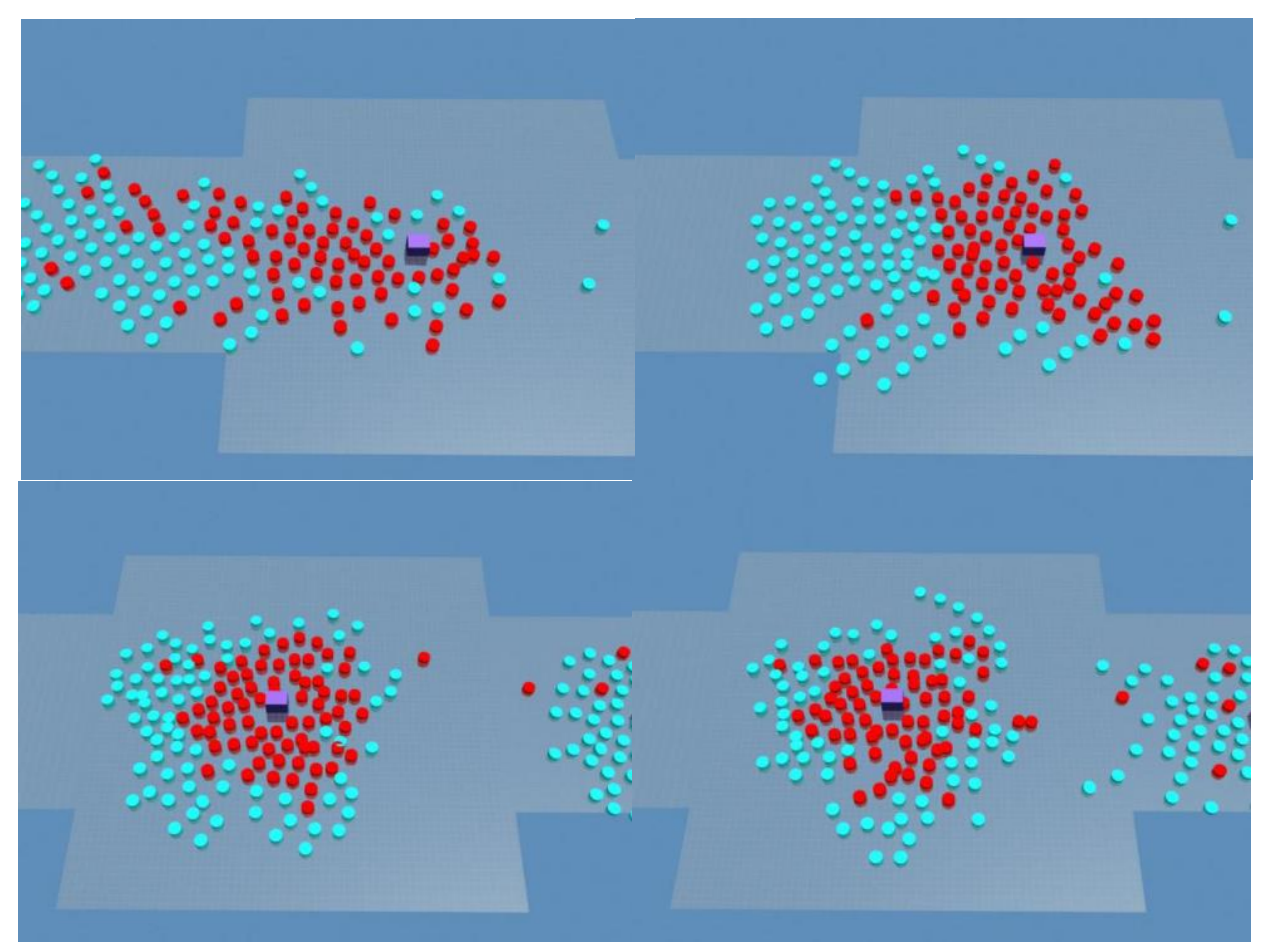

Figure 8. Simulation results with infector degeneration.

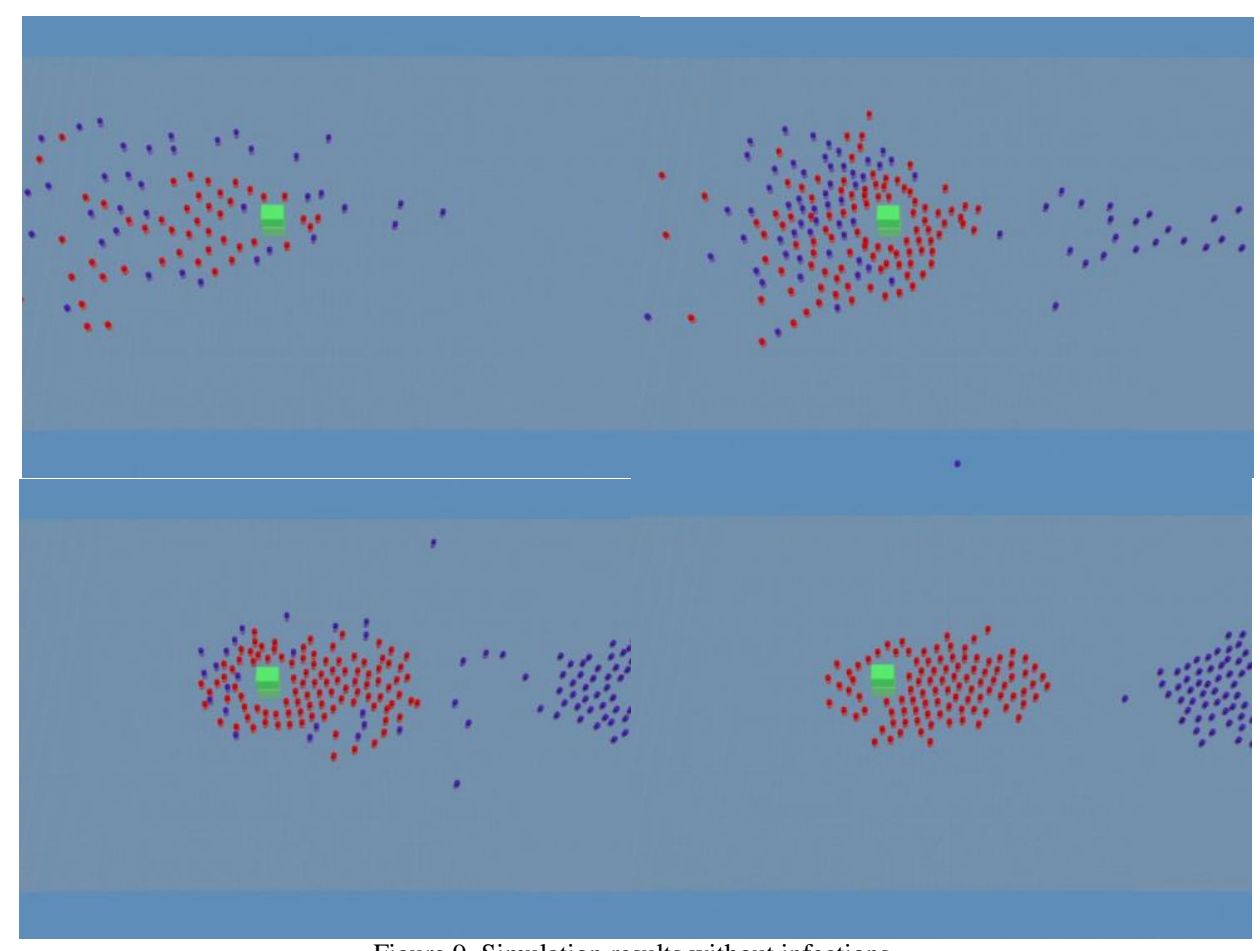

Figure 9. Simulation results without infections.

Actually, the case in Figure 8 often happens in our daily life when customers walk alone on a business street. He or she may be attracted by the propaganda of the shop and move to that shop. His or her movements affected other customers who 
may also go to that shop. After he or she discovers the shop's information, he or she would lose interest and change into immune. Figure 10 represents the emotional affections on individuals' decision making.

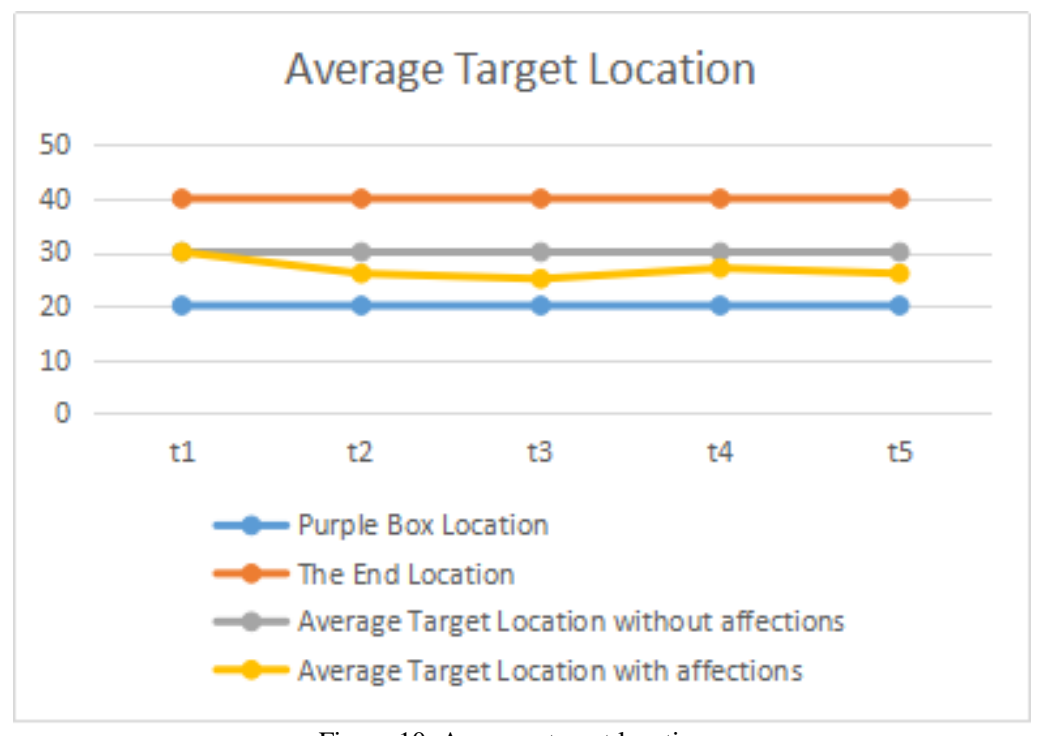

Figure 10. Average target locations.

According to Figure 10, we can find that because of the emotional affections of individuals, whose original target was the end, changed their target into the purple box, the average target location was smaller than 30 . In time t4, the average target location increased because of the degeneration.

\section{Conclusions}

In this paper, we provided a SIR model based emotion contagion simulation method. Similar to our previous works, each individual was represented by a square and was computed independently so that the positions of individuals can be changed dynamically. We treated the emotion contagion process as the information spreading process so that the SIR model could be adopted to simulate the emotion contagion. As a consequence, the degeneration situation could be easily integrated into our simulation. Our future works should develop an empirical degeneration formula that is more suitable to the real crowd rather than the social network formula.

\section{Acknowledgements}

This research is supported by National Natural Science Foundation of China (No. 61502064 and No. 61502065). The Fundamental Science and Frontier Technology Research Projects of Chongqing (No. cstc2017jcyjAX0089, No. cstc2017jcyjBX0059).

\section{References}

1. T. Bosse, R. Duell, Z. A. Memon, et al, “Agent-Based Modeling of Emotion Contagion in Groups,” Cognitive Computation, vol. 7, no. 1, pp. 111-136, 2015

2. A. A. Bakar, M. A. Majid, K. Hammad, "An Overview of Crowd Evacuation Simulation,” Advanced Science Letters, vol. 4, no. 2, pp. 400-407, 2016

3. T. Bosse, M. Hoogendoorn, M. C. Klein, et al, "Modelling Collective Decision Making in Groups and Crowds: Integrating Social Contagion and Interacting Emotions, Beliefs and Intentions," Autonomous Agents and Multi-Agent Systems, vol. 27, no. 1, pp. 52-84, 2013

4. D. Centola, "The Spread of Behavior in an Online Social Network Experiment," Science, vol. 329, no. 5996, pp. 1194-7, 2010

5. J. Gratch, L. Cheng, S. Marsella, "The Appraisal Equivalence Hypothesis: Verifying The Domain-Independence of a Computational Model of Emotion Dynamics," International Conference on Affective Computing and Intelligent Interaction. IEEE, pp. 105-111, 2015

6. S. Kim, S. J.Guy, D. Manocha, et al, "Interactive Simulation of Dynamic Crowd Behaviors Using General Adaptation Syndrome Theory," ACM SIGGRAPH Symposium on Interactive 3d Graphics and Games, pp. 55-62, 2012

7. Z. Meng, X. Fu, "Dynamic Information Spreading Model Based on Online Social Network," Journal of Computer Applications, vol. 34, no. 7, pp. 1960-1963, 2014 
8. F. Martinez-Gil, M. Lozano, F. Fernández, "Strategies for Simulating Pedestrian Navigation with Multiple Reinforcement Learning Agents," Autonomous Agents and Multi-Agent Systems, vol. 29, no. 1, pp. 98-130, 2015

9. F. Martinez-Gil, M. Lozano, F. Fernández, "MARL-Ped: A Multi-Agent Reinforcement Learning Based Framework to Simulate Pedestrian Groups," Simulation Modelling Practice \& Theory, vol. 47, no. 47, pp. 259-275, 2014

10. Y. Moreno, M. Nekovee, A. F. Pacheco, "Dynamics of Rumor Spreading in Complex Networks," Physical Review E Statistical Nonlinear \& Soft Matter Physics, vol. 69, pp. 066130, 2004

11. S. Navlakha, C. Faloutsos, Z. Bar-Joseph, "MassExodus: Modeling Evolving Networks in Harsh Environments," Data Mining \& Knowledge Discovery, vol. 29, no. 5, pp. 1211-1232, 2015

12. M. Nekovee, Y. Moreno, G. Bianconi, et al, "Theory of Rumour Spreading in Complex Social Networks," Physica A Statistical Mechanics \& Its Applications, vol. 374, no. 1, pp. 457-470, 2007

13. A. A. Ojugo, B. E. Iwhiwhu, D. Kekeje, et al, "Malware Propagation on Social Time Varying Networks: A Comparative Study of Machine Learning Frameworks," International Journal of Modern Education \& Computer Science, vol. 6, no. 8, 2014

14. B. A. Prakash, H. Tong, N. Valler, et al, "Virus Propagation on Time-Varying Networks: Theory and Immunization Algorithms," American Journal of Medicine, vol. 73, no. 1A, pp. 300-4, 2010

15. D. P. Papadopoulos, "Cellular Automaton Model of Crowd Evacuation Inspired by Slime Mould," International Journal of General Systems, vol. 44, no. 3, pp. 354-391, 2015

16. J. E. Steephen, "HED: A Computational Model of Affective Adaptation and Emotion Dynamics," IEEE Transactions on Affective Computing, vol. 4, no. 2, pp. 197-210, 2013

17. M. R. Sanatkar, W. N. White, B. Natarajan, et al, "Epidemic Threshold of an SIS Model in Dynamic Switching Networks," IEEE Transactions on Systems Man \& Cybernetics Systems, vol. 46, no. 3, pp. 345-355, 2016

18. L. Tan, M. Hu, H. Lin, "Agent-based Simulation of Building Evacuation: Combining Human Behavior with Predictable Spatial Accessibility in a Fire Emergency," Information Sciences, vol. 295, pp. 53-66, 2015

19. D. Thalmann, S. R. Musse, "Crowd Simulation," Springer Publishing Company, Incorporated, 2012

20. N. Xiang, Z. Pan, L. Zhu, et al, "Dynamic Crowd Emotion Contagion Simulation with GPU Acceleration," International Conference on Cyberworlds, IEEE Computer Society, pp. 175-178, 2016

21. X. Yang, H. Dong, Q. Wang, et al, "Guided crowd dynamics via modified social force model," Physica A Statistical Mechanics \& Its Applications, vol. 411, no. 10, pp. 63-73, 2014

22. W. Zeng, H. Nakamura, and P. Chen, "A Modified Social Force Model for Pedestrian Behavior Simulation at Signalized Crosswalks," Procedia - Social and Behavioral Sciences , vol. 138, pp. 521-530, 2014

23. B. Zhou, X. Tang, X. Wang, "Learning Collective Crowd Behaviors with Dynamic Pedestrian-agents," International Journal of Computer Vision, vol. 111, no. 1, pp. 50-68, 2015

24. L. Zhao, J. Wang, Y. Chen, et al, "SIHR Rumor Spreading Model in Social Networks," Physica A Statistical Mechanics \& Its Applications, vol. 391, no. 7, pp. 2444-2453, 2012

25. D. Zhao, J. Wang, X. Zhang, et al, "A Cellular Automata Occupant Evacuation Model Considering Gathering Behavior," International Journal of Modern Physics C, vol. 26, no. 08, pp. 1550089, 2015

26. D. Zhao, B. Yegenmammedov, P. Liu, et al, "Comparative Study on Occupant Evacuation with Building EXODUS and a Cellular Automaton Model,” Open Journal of Safety Science \& Technology, vol. 07, no. 1, pp. 42-57, 2017

Xiang Nan is currently an associate professor of Liangjiang International College, Chongqing University of Technology. He received his Bachelor degree from Xi'an Jiaotong University in 2006, his Master degree from Chongqing University of Posts and Telecommunications in 2009 and his PhD from State Key Lab of CAD\&CG, Zhejiang University in 2013. He visited Korea Advanced Institute of Science and Technology, as a visiting scholar in 2015. Now he is the head of computer science and technology department, Liangjiang International College, Chongqing University of Technology. His current research interests include virtual reality, affective computing and collective intelligence.

Zhou Zehong is a master student from the School of Computer Science and Engineering, Chongqing University of Technology. His research interests include artificial intelligence and machine learning.

Pan Zhigeng is currently the professor of Institute of Service Engineering, Hangzhou Normal University. He is the managing director and deputy secretary-general of Chinese Association for Image and Graphics; secretary-general of Virtual Reality Special Committee; deputy director of the Special Committee of Machine Perception and Virtual Reality, Chinese Association for Artificial Intelligence. He is also the editorial board member of International Journal of Image and Graphics (IJIG) and International Journal of CAD/CAM. 